Check for updates

Cite this: RSC Adv., 2017, 7, 20068

Received 10th January 2017

Accepted 20th March 2017

DOI: $10.1039 / c 7 r a 00380 c$

rsc.li/rsc-advances

\title{
Small water-soluble pyrimidine hexafluorophosphate derivatives with high two- photon absorption activities in the near-IR region and their biological applications $\uparrow$
}

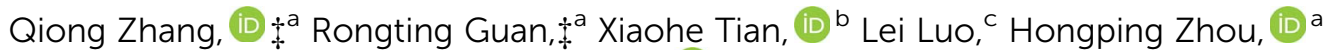
Shengli $\mathrm{Li}^{a}{ }^{a}$ Jieying $\mathrm{Wu}^{\star a}$ and Yupeng Tian (D) *a

Small, biocompatible and water-soluble molecules with moderate two-photon absorption (2PA) crosssection values $(\sigma)$ are in extreme demand for specific bioimaging applications. Herein, two novel imidazole-pyrimidine hexafluorophosphate derivatives (6P and 10P) are efficiently synthesized through improved Knoevenagel condensation and Ullmann reactions with high yield. Based on systematic photophysical investigations and theoretical calculations, the structure-property relationships indicate that the different donor groups have large influences on their optical properties. The 2PA cross-section values $(\sigma)$ are obtained both by Z-scan and two-photon excited fluorescence (2PEF) measurements. It is found that the two-photon absorption cross section values $(\sigma)$ in the near-infrared region are significantly enhanced for $6 \mathrm{P}$ and $10 \mathrm{P}$, which confirms that these small molecules display a suitable turnON fluorescence response for two-photon fluorescence microscopy (2PFM). Comprehensively considering the high solubility of the introduced hexafluorophosphate group, 6P and 10P possess high specificity for mitochondrial localization, showing a temperature-independent pathway, possibly passive infusion, which is advantageous in comparison with the commercially available mitochondrial trackers. Due to their low cytotoxicity, these small molecules offer a promising platform to directly monitor the intestinal system in live zebrafish larvae.

\section{Introduction}

Two-photon excitation (TPE) has particular practical significance because of the development of highly efficient two-photon absorbing (TPA) materials and their applications. ${ }^{1}$ The recent technologies that can exploit small molecular materials with the two-photon absorption (2PA) property have significantly been applied in the areas of chemistry, biology and photonics, such as three-dimensional micro-fabrication, high capacity data storage, ${ }^{2}$ optical limiting, two-photon laser scanning fluorescence imaging, ${ }^{3}$ and photodynamic therapy. ${ }^{4}$ Therefore, considerable research efforts have been devoted to develop novel small molecule materials with strong 2PA activity for the last decade. One of these important applications is the two-photon fluorescence imaging ${ }^{5,6}$ of living cells, which includes the imaging of various

\footnotetext{
${ }^{a}$ Department of Chemistry, Key Laboratory Inorganic Material Chemistry of Anhui Province, Anhui University, Hefei 230039, PR China. E-mail: zhangqiong.314@163. com; jywu1957@163.com; yptian@ahu.edu.cn

${ }^{b}$ School of Life Science, Anhui University, 230601, Hefei, P. R. China

${ }^{c}$ College of Pharmaceutical Science, Southwest University, 400715, China

$\dagger$ Electronic supplementary information (ESI) available. See DOI: $10.1039 / \mathrm{c} 7 \mathrm{ra00380 \textrm {c }}$

$\ddagger$ These authors contributed equally to this work.
}

organelles, such as the lysosome, ${ }^{7}$ endoplasmic reticulum, ${ }^{8}$ Golgi apparatus, ${ }^{9}$ and mitochondria. ${ }^{10}$

It should be noted that pyrimidine derivatives have been intensively investigated as nonlinear optical materials ${ }^{11-14}$ owing to the planarity they provide. Recently, several TPA chromophores utilizing pyrimidine as an electron-withdrawing central core substituent have been synthesized, and fairly good TPA cross section values have been measured for them..$^{15}$ Moreover, pyrimidine is found in various natural products that are the important constituents of a number of modern drugs. It exhibits adequate safety, tolerability and clinical efficacy profiles in preclinical and clinical trials. ${ }^{16}$ The introduction of flexible ether chains and ethoxyphenyl triphenylamine can not only increase the solubility of this molecule, but also enhance the electron-donating property of the amino group. On the other side, an issue of primary importance is the solubility of products in several common organic solvents and water. Herein, we demonstrate that hexafluorophosphate can be solubilized in DMSO/water.

Considering the abovementioned design strategy and based on our previous study, ${ }^{15,16}$ a series of new $\mathrm{D}-\pi-\mathrm{A}$ type 2 imidazolyl-4(6)methyl-pyrimidine hexafluorophosphates (6P and $10 \mathrm{P}$ ) is designed (Fig. 1). The 2PA cross-section values $(\sigma)$ are obtained both by Z-scan and two-photon excited 


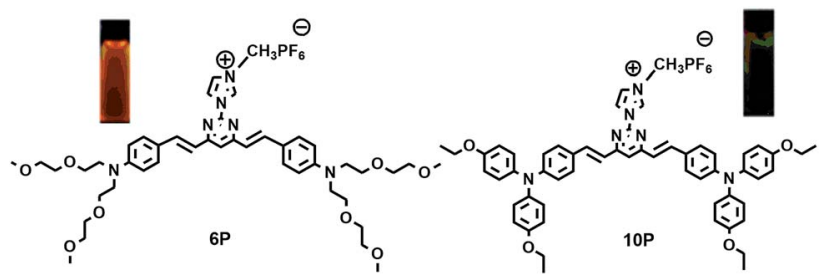

Fig. 1 Molecular structures of $6 \mathrm{P}$ and $10 \mathrm{P}$

fluorescence (2PEF) measurements. The N,N-bis(4-ethoxyphenyl)aniline groups and ethoxyphenyl triphenylamine groups have rich $\pi$-electron densities. The imidazolium and hexafluorophosphate groups can increase the water solubility of the molecules in DMSO- $\mathrm{H}_{2} \mathrm{O}$ systems, as well as enhance the extent of electron delocalization and ability of the 2PA chromophore to accept electrons. This, in turn, enables the use of biological-friendly solvents (e.g. PBS) in later long-term live cell experiments. ${ }^{17}$ Relying on their comprehensive studies, $6 \mathrm{P}$ and $10 \mathrm{P}$ were selected for a bioimaging study using two-photon scanning microscopy. The bioimaging results show that $6 \mathrm{P}$ and $10 \mathrm{P}$ would target mitochondria with monitoring of the fluorescence signals for a long period of time (0-150 s), and they are capable of specifically monitoring the fluorescent signals in the intestinal system of living zebrafish. These results suggest that $6 \mathrm{P}$ and $10 \mathrm{P}$ could be potentially applied as $2 \mathrm{PA}$ probes for in vivo and in vitro imaging.

\section{Results and discussion}

\section{Synthesis}

As shown in Fig. 1 and Schemes S1 and S2, $\dagger$ 6P and 10P were prepared by the Ullmann reaction using imidazole and 2iodide-4-methyl-pyrimidine et al. in the presence of $t$-BuOK, which afforded the title product in over $80 \%$ yield. ${ }^{15}$ EX- 6 and EX-10 were synthesized by the Witting reaction. ${ }^{15,16}$ The details of the synthesis and characterization data are given in the Experimental section. All compounds were characterized by elemental analyses, IR, MOLDI-TOF-MS and NMR spectral techniques.

\section{Theoretical calculations}

Fig. 2 gives straightforward representations of the electron density distribution of $10 \mathrm{P}$. Orbital analysis exhibits that the highest occupied molecular orbital (HOMO) is comprised of $\pi$ orbitals localized on the benzene and vinyl groups. Moreover, the lowest unoccupied molecular orbital (LUMO) is localized in the pyrimidine orbitals with abundant conjugated $\pi$-bridges. Thus, there are relatively strong $\pi$-donor interactions between the ethoxyphenyl triphenylamine group and the pyrimidine center. As shown in Fig. 2 and 3, the lowest energy absorption band can be assigned to ICT between the nitrogen atom of the amino group and the pyrimidine ring. Basically, the calculated singlet-singlet transitions in 10P are in reasonable agreement with the experimental $\lambda_{\mathrm{ab}}$ in the absorption bands observed (Fig. 4).

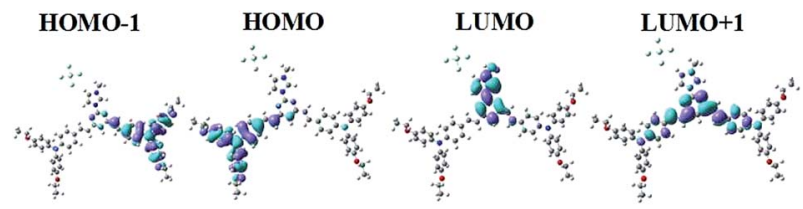

Fig. 2 Representation of the calculated HOMO and LUMO orbitals of $10 \mathrm{P}$

\section{Linear absorption and single-photon excited fluorescence (OPEF)}

The photophysical properties of all the compounds are summarized in Table 1 . The linear absorption spectra of 6P and 10P are shown in Fig. 3(a), from which it can be seen that 6P and 10P exhibit low energy bands between 400 and $450 \mathrm{~nm}$; they originate from the $\pi-\pi^{*}$ transition and mix with the intramolecular charge transfer (ICT), as confirmed by TD-DFT calculations (Fig. 2). As an example, TD-DFT computational studies were performed to further explain the electronic structures of the ground state of 10P. The TD-DFT $\{[6-31 G(d)]\}$ calculations indicate that the low energy transition presents marked charge transfer (CT) character, with the HOMO and the LUMO being mainly located on the flexible ether chains (denoted as $\pi_{\mathrm{D}}$ ) and phenyl unit (denoted as $\tau_{\text {phenyl }}^{*}$ ) with moderate contribution from the pyrimidine-imidazole group and vinyl moiety (denoted as $\pi_{\mathrm{A}}^{*}$ ), respectively. The remaining band $(\lambda=390.61 \mathrm{~nm}, f=0.28)$ is assigned mainly to the $\pi_{\mathrm{D}} \rightarrow \pi_{\mathrm{A}}^{*}$ transition as a result of the HOMO-1 $\rightarrow$ LUMO transition with some contributions from an ICT transition $\left[\pi_{\mathrm{D}} \rightarrow \pi_{\text {phenyl }}^{*}\right.$, $\mathrm{HOMO}-1 \rightarrow$ LUMO+1].

As shown in Fig. 3, weak solvatochromism is observed in the absorption bands, which indicates that there is a slight difference in dipoles between the ground state and excited state of the chromophores. Moreover, the maximum absorption bands $\left(\lambda_{\max }\right)$ of the compounds, shown in Fig. 3(a), display slightly negative solvatochromism because of the opposite polar forms in the ground and excited state. Upon increasing the solvent polarity, the fluorescence spectra of 10P show a red shift, together with a monotonically increasing tendency in the Stokes shift, which can be explained by the fact that the excited state may possess higher polarity than the ground state since solvatochromism is associated with a lowering of the energy levels, and an increased dipoledipole interaction between the solute and solvent leads to symmetry breaking (Fig. 3(b) and Table 1). In addition, 6P exhibits strong fluorescent intensity compared with $10 \mathrm{P}$ by changing from a flexible ether oxygen chain to the ethoxyphenyl triphenylamine group, which resulted from the fact that $N, N$-bis(4-ethoxyphenyl)aniline group (rather than ethoxyphenyl triphenylamine group), owing to delocalization of the $\pi$ electrons onto the $N, N$-bis(4-ethoxyphenyl)aniline, as an appropriate electron-donating group can improve the ICT. The stronger donor and acceptor group combined together in $10 \mathrm{P}$ may cause an energy loss in the excited state vibration to quench the fluorescence, resulting in a lower fluorescence quantum yield compared with $6 \mathrm{P} .{ }^{\mathbf{1 8 , 1 9}}$ 

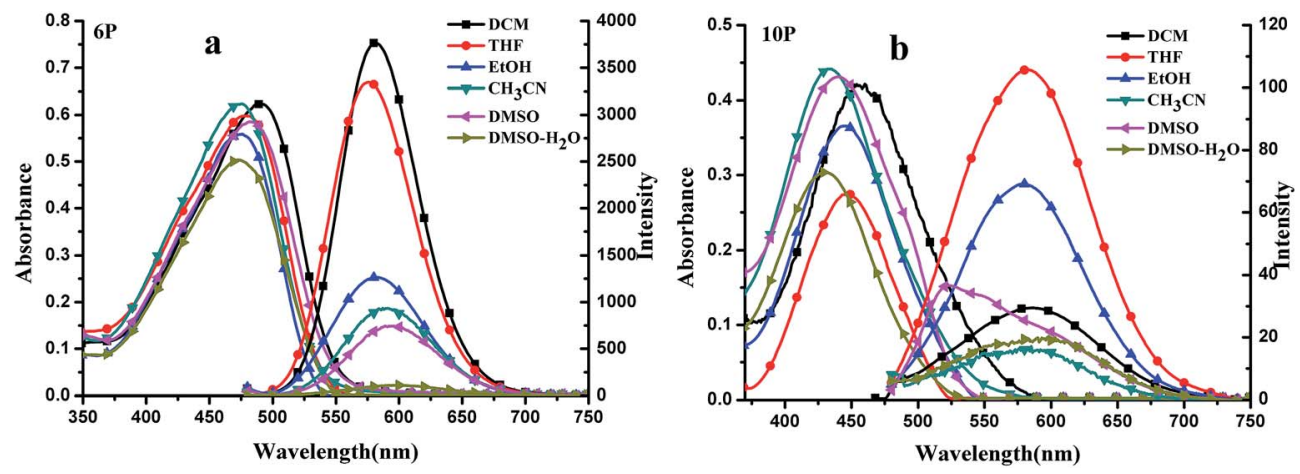

Fig. 3 ( $a$ and b) UV-Vis and OPEF spectra of $6 \mathrm{P}$ and 10P $\left(c=1.0 \times 10^{-5} \mathrm{~mol} \mathrm{~L}^{-1}\right)$ in six organic solvents.

Diluted solutions $\left(5 \times 10^{-6} \mathrm{M}\right)$ of $6 \mathrm{P}$ and $10 \mathrm{P}$ in MeTHF from 80-200 K show analogous emission bands that are particularly sensitive to the excitation wavelength. As the temperature increases from 80 to $200 \mathrm{~K}$, there is a gradual emission intensity decrease. This emission spectral change can be reversed by cooling the solution from room temperature to $80 \mathrm{~K}$. At $80 \mathrm{~K}$, the yellow-green emission is vibronically structured, with peak maxima at $578(6 \mathrm{P})$ and $559 \mathrm{~nm}(10 \mathrm{P})$, upon reducing the temperature from 220 to $80 \mathrm{~K}$, the yellow-green emission increases and a blue shift $\left(\lambda_{\max }=575 \mathrm{~nm}, 10 \mathrm{P}\right)$ becomes apparent. The increase in the intensities of the bands upon lowering the temperature implies that there is a thermally activated non-radiative decay pathway that becomes less significant at reduced temperatures. ${ }^{20} \mathrm{At}$ temperatures above $140 \mathrm{~K}, 6 \mathrm{P}$ and $10 \mathrm{P}$ are non-emissive in 2-MeTHF fluid solution, presumably due to the efficient non-radiative decay arising from the molecular motion of the flexible ether oxygen chain to the ethoxyphenyl triphenylamine group. The non-radiative decay decreases as the temperature decreases, and at temperatures below $140 \mathrm{~K}$, both yellow-green emissions start to develop in the more polar 2MeTHF glassy solutions. We suggest that the non-radiative decay of the $3 \pi \pi^{*}$ excited state has a stronger temperature dependence than that of the excimeric $3 \pi \pi^{*}$ excited state. ${ }^{21}$

\section{Fluorescence lifetime and quantum yield}

To gain more insight into the radiative and nonradiative decay processes of $6 \mathrm{P}$ and 10P, we also conducted lifetime experiments (Table 1). With an increase in the polarity of the solvents, the fluorescence lifetimes of the compounds increase generally. This can be explained by the stronger solute/solvent interaction at the excited state compared with that at the ground state, which indicates that the increasing polarity of the excited state increases. The energy level can be lowered greatly by increasing the dipole-dipole interaction between the solute and solvent. The quantum yields $(\Phi)$ of the compounds $6 \mathrm{P}$ and $10 \mathrm{P}$ in different solvents were determined using fluorescein as a standard. As shown in Table 1, the quantum yields of $10 \mathrm{P}$ decrease generally as the polarity of the solvent increases except in EtOH and DMSO. Note that the quantum yields of $10 \mathrm{P}$ obtained in EtOH are smaller than that in DMSO because of the negative solvatochromism effect due to opposite polar forms in the ground and excited states. ${ }^{22}$ On the contrary, for $6 \mathrm{P}$, the quantum yield decreases generally with solvent polarity, exhibiting a positive solvato-kinetic effect, which could be explained by the fact that the hydrogen bonds consume some energy from the excited state molecules.

\section{Two-photon excited fluorescence (2PEF) and two-photon absorption (2PA)}

As shown above, moderate fluorescence was detected in 6P; however, 10P may cause energy loss in the excited state vibration to quench the fluorescence. Accordingly, we have investigated the two-photon absorption properties of $6 \mathrm{P}$ and $10 \mathrm{P}$ by using $2 \mathrm{PEF}$ and Z-scan methods.
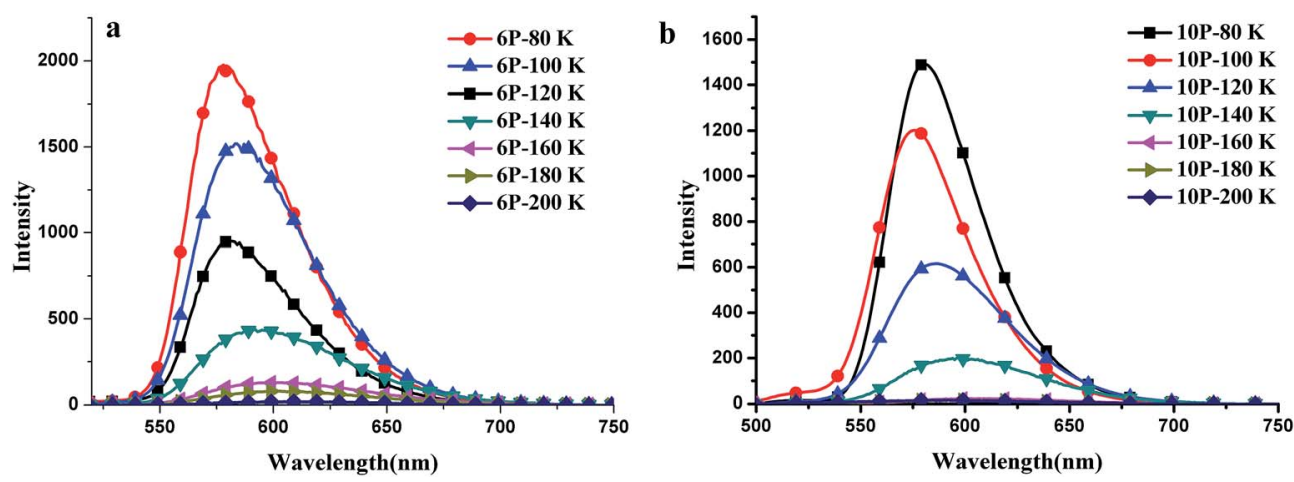

Fig. 4 ( $\mathrm{a}$ and b) OPEF spectra of $6 \mathrm{P}$ and $10 \mathrm{P}\left(\mathrm{c}=5.0 \times 10^{-6} \mathrm{~mol} \mathrm{~L}^{-1}, \mathrm{MeTHF}\right)$ from $80-200 \mathrm{~K}$. 
Table 1 Photophysical properties of 6P and 10P in six different polar solvents

\begin{tabular}{lllllll}
\hline & Solvents & $\lambda_{\max }{ }^{a}$ & $\varepsilon_{\max }{ }^{b}$ & $\lambda_{\max }{ }^{c}$ & $\Phi^{d}$ & $\tau[\mathrm{ns}]$ \\
\hline \multirow{6}{*}{$6 \mathrm{P}$} & & 489 & 6.32 & 580 & 0.43 & 1.63 \\
& DCM & 479 & 6.00 & 575 & 0.42 & 2.08 \\
& $\mathrm{THF}$ & 475 & 5.64 & 578 & 0.19 & 0.79 \\
& $\mathrm{EtOH}$ & 474 & 6.20 & 588 & 0.12 & 0.27 \\
& $\mathrm{CH}_{3} \mathrm{CN}$ & 483 & 5.92 & 590 & 0.10 & 0.87 \\
& $\mathrm{DMSO}$ & 472 & 5.00 & 593 & 0.03 & 1.34 \\
& $\mathrm{DMSO}-\mathrm{H}_{2} \mathrm{O}$ & 452 & 3.97 & 549 & 0.029 & 0.88 \\
& $\mathrm{DCM}$ & 450 & 4.52 & 548 & 0.021 & 0.97 \\
& $\mathrm{THF}$ & 446 & 4.91 & 559 & 0.024 & $<0.05$ \\
& EtOH & 434 & 4.66 & 585 & 0.021 & 0.25 \\
& $\mathrm{CH}{ }_{3} \mathrm{CN}$ & 440 & 5.21 & 559 & 0.025 & 1.36 \\
& $\mathrm{DMSO}$ & 428 & 4.27 & 592 & 0.019 & 1.71
\end{tabular}

${ }^{a}$ Absorption peak position in $\mathrm{nm}\left(1 \times 10^{-5} \mathrm{~mol} \mathrm{~L}^{-1}\right) \cdot{ }^{b}$ Maximum molar absorbance in $10^{4} \mathrm{~mol}^{-1} \mathrm{~L} \mathrm{~cm}^{-1}$. ${ }^{c}$ Peak position of OPEF in $\mathrm{nm}(1.0 \times$ $\left.10^{-5} \mathrm{~mol} \mathrm{~L}^{-1}\right)$, excited at the absorption maximum. ${ }^{d}$ Quantum yields determined by using coumarin $307(\Phi=0.56)\left(1.0 \times 10^{-5} \mathrm{~mol} \mathrm{~L}^{-1}\right)$ as the standard.

\section{Two-photon excited fluorescence (2PEF)}

The two-photon fluorescence spectra of $6 \mathrm{P}$ in six organic solvents with $c=0.1 \mathrm{mM}$ were measured from 700 to $900 \mathrm{~nm}$ with an interval of $10 \mathrm{~nm}$ and a pulse duration of $140 \mathrm{fs}$ under $300 \mathrm{~mW}$. Fig. S1† shows the $\log -\log$ plot of the excited fluorescence signal versus excited light power. It provides direct evidence for the squared dependence of excited fluorescence power and input laser intensity upon excitation with a tunable property in this range. Therefore, it should be surely assigned to $2 \mathrm{PEF}$. In Fig. $\mathrm{S} 2$ and $\mathrm{S} 3, \dagger$ the $2 \mathrm{PEF}$ spectra of $6 \mathrm{P}$ exhibit a broad emission band with an evident red shift compared to its OPEF spectra, which can be explained by the re-absorption effect. The short wavelength side of the two-photon fluorescence was reabsorbed by the solution and red-shifts of the fluorescence spectra were readily observed. ${ }^{23}$

The action 2PA cross sections $(\Phi \sigma)$ of $6 \mathrm{P}$ in different solvents (Fig. 5) show that the largest action 2PA cross sections of $6 \mathrm{P}$ in various solvents are all located in the NIR region, and $6 \mathrm{P}$ has the largest action 2PA cross section in DCM ( 200 GM) and also a moderate value in DMSO ( $90 \mathrm{GM})$ at the wavelength of $780 \mathrm{~nm}$. Clearly, the polarity of the solvent and the excitation wavelength has a significant influence on the action two-photon absorption cross section. Compared to the previous chromophores derived from pyrimidine, ${ }^{15,16,24,25}$ small changes in the structure may have important implications in terms of molecular engineering for specific applications because the 2PA properties (in terms of cross sections, position of the maximum, and bandwidth), as well as the one photon and the photoluminescence characteristics can be affected considerably. Increasing the branch number results in a pronounced enhancement of the 2PA cross sections in the NIR region, which may imply that extending the $\pi$-conjugation domain is accomplished by increasing the number of branches. The pyrimidine molecule based on a multi-branched vinylene could be a useful approach toward enhanced molecular 2PA properties. On the other hand, comparison of the 2PA properties

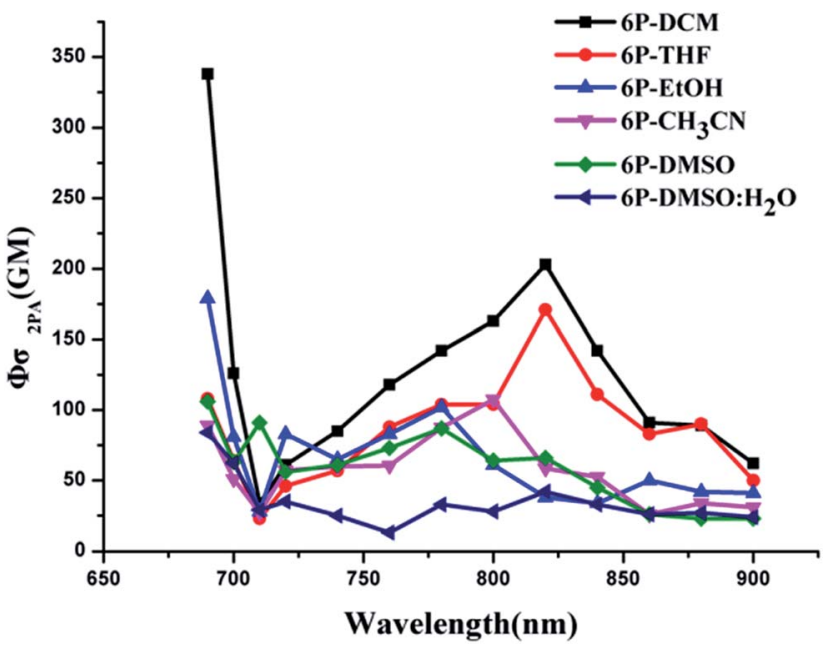

Fig. 5 Action 2PA cross section spectra of $6 \mathrm{P}$ at the optimal excitation wavelength from $700-920 \mathrm{~nm}$ with $c=0.1 \mathrm{mM}$ in six organic solvents.

reveals the relative efficiency of the cores. The values of $\Phi \sigma$ for $6 \mathrm{P}$ are smaller than $\mathrm{L} 1,{ }^{16}$ understandably due to the acceptors linked to the cores. $\Phi \sigma$ decreases by ten times when the core is changed from imidazolyl to imidazolyl-hexafluorophosphate. This indicates that the relative efficiency of the core in the $\mathrm{D}-\pi-\mathrm{A}$ quadrupoles decreases in the order, imidazolyl > imidazolylhexafluorophosphate, which is less prone to the ICT progress in the D- $\pi-\mathrm{A}$ system, leading to weak $2 \mathrm{PA}$ activity.

\section{Third-order nonlinear optical properties (two-photon absorption (2PA))}

Because 10P has no nonlinear absorption in DMSO, only its third-order optical nonlinearities of are presented. The excitation wavelength of $10 \mathrm{P}$ is $730 \mathrm{~nm}$. The open aperture Z-scan curves are shown in Fig. 6(a). Based on the Z-scan equations (in the ESI $\dagger$ ), the values of $\sigma$ for 10P are given in Fig. 6(b). Clearly, the 2PA cross section values $\sigma$ of $10 \mathrm{P}$ are $15171 \mathrm{GM}$ (DCM), $4428 \mathrm{GM}$ (THF), $15641 \mathrm{GM}(\mathrm{EtOH}), 847 \mathrm{GM}\left(\mathrm{CH}_{3} \mathrm{CN}\right)$, $951 \mathrm{GM}$ (DMSO) and $266 \mathrm{GM}$ (DMSO : $\mathrm{H}_{2} \mathrm{O}$ ). These results reveal that $10 \mathrm{P}$ exhibits a discernible TPA spectral feature in different solvents, particularly in EtOH solution. Such a difference must be due to the varied substituent group of the pyrimidine-based chromophores, which may arise from the strong electron-donating and withdrawing effect of 10P. The solvent-dependence of the two-photon absorption cross section value was reported by Bazan et al. ${ }^{26}$ and our group. ${ }^{27}$ Generally, the maxima TPA cross-section values are observed in a solvent with intermediate polarity. ${ }^{28}$ Considering the remarkable value of $\sigma$, which is caused by its highly delocalized $\pi$-electronic configuration and large intrinsic polarizability, 10P is a good candidate as a third-order nonlinear optical material. Importantly, the strong third-order nonlinear optical response occurs within the near-infrared region, which encouraged us to further investigate its applications in biology.

We should point out that there was still an observed difference in the absolute values of the two-photon cross sections $(\sigma)$ 

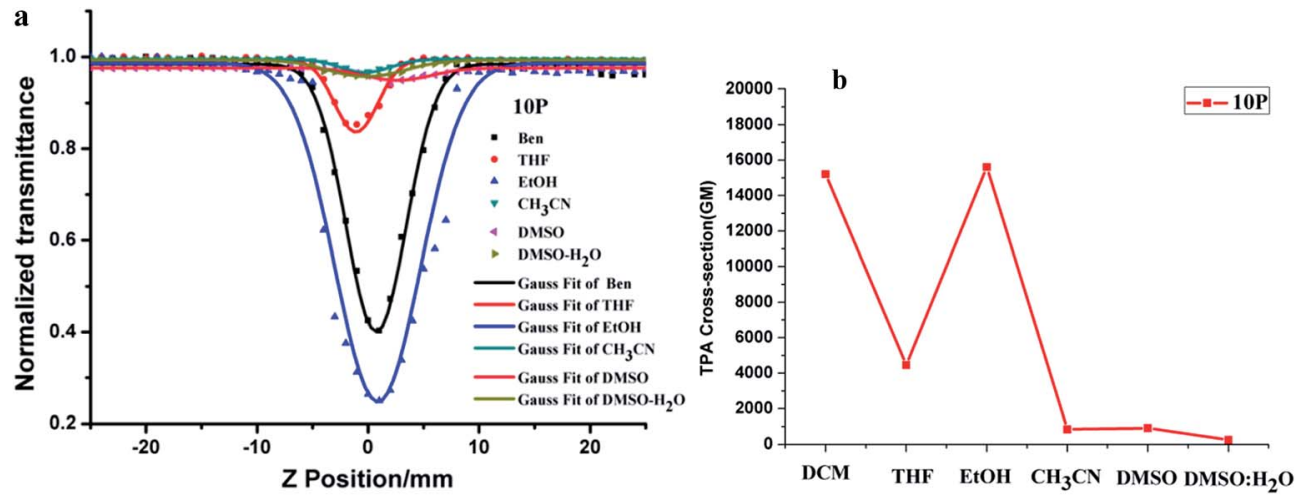

Fig. 6 (a) Normalized open aperture Z-scan curves of 10P $\left(\lambda_{\mathrm{ex}}=730 \mathrm{~nm}\right)$ in six organic solvents with $c=0.1 \mathrm{mM}$. The filled squares represent the experimental data and the solid curve is the theoretical data. (b) TPA spectra of 10P at the optimal excitation wavelength $\lambda_{\mathrm{ex}}=730 \mathrm{~nm}$ with $c=$ $0.1 \mathrm{mM}$ in six organic solvents.

obtained from the two techniques. The Z-scan method yields $\sigma$ directly from the experimental measurement, whereas the twophoton fluorescence technique results in a quantity, twophoton excited action crosssection $(\Phi \sigma)$, which is simply the product of absolute $\sigma$ and the single-photon fluorescence quantum efficiency. Thus, the two photon cross section values obtained by the Z-scan method could be overestimated compared to that obtained by the two-photon excited fluorescence method due to the fact that no influence of fluorescence quantum yield was considered in the measurement. ${ }^{29}$ However, the results of $\sigma$ values obtained by the Z-scan method have a magnitude equal to that of the values obtained by the twophoton excited fluorescence method. The reason for this may be attributed to the fact that an fs laser was used as the light source. Nevertheless, the trend of $\sigma$ values observed for these chromophores should still be valid.

With the abovementioned findings, $6 \mathrm{P}$ and $10 \mathrm{P}$ bearing the modified imidazole-pyrimidine hexafluorophosphate unit exhibit moderate two-photon cross sections located the near IR region in highly polar solvent and good solubility in the DMSO$\mathrm{H}_{2} \mathrm{O}$ system, which encouraged us to further study their potential application in bioimaging.

\section{Cell imaging and zebrafish imaging}

MTT assay. Since high cell viability is essential for biological applications, a cytotoxicity test was run via the 3-(4,5dimethylthiazol-2-yl)-2,5-diphenyltetrazilium bromide (MTT) assay in HepG2 cells. Fig. S4 $\uparrow$ demonstrates that after over 24 hours incubation with $6 \mathrm{P}$ and 10P, high cell viability is obtained at concentrations between $20 \mu \mathrm{M}$ and $100 \mu \mathrm{M}$. This result suggests that $6 \mathrm{P}$ and 10P exhibit low toxicity in living cells.

Cellular uptake properties. Cellular uptake is critical to the success of a compound as an intracellular probe. HepG2 cells, as a model, were incubated with $20 \mu \mathrm{M} 6 \mathrm{P}$ and $10 \mathrm{P}$ for $30 \mathrm{~min}$. The cells were then imaged using confocal microscopy after washing with PBS $(\times 3)$. Fig. S5 $\uparrow$ shows that $6 \mathrm{P}$ and $10 \mathrm{P}$ penetrate into the cell cytosol within a short incubation period and display intensive luminescence in both one-photon and twophoton channels; the signal is uniformly localized in the cytosolic OPAce and it is apparently excluded from the nuclear region. The bright field micrographs show a good cell shape after cellular internalization, which highly implies the toxicity of $6 \mathrm{P}$ and $10 \mathrm{P}$.

A co-staining experiment using MitoTracker was performed to further determine whether $6 \mathrm{P}$ and $10 \mathrm{P}$ were internalized in membrane-rich organelle mitochondria (Fig. 7). The fluorescence of $6 \mathrm{P}$ and $10 \mathrm{P}$ overlap with that of MitoTracker with a Pearson's coefficient $\mathrm{Rr}=0.992$ and 0.998 , respectively. This suggests that $6 \mathrm{P}$ and $10 \mathrm{P}$ localize to the mitochondria, which is essential for mitochondrial targeting probes.

To determine the possible mechanism of $6 \mathrm{P}$ and $10 \mathrm{P}$ cell entry, HepG2 cells were incubated with $20 \mu \mathrm{M} 6 \mathrm{P}$ and 10P for $30 \mathrm{~min}$ at $4{ }^{\circ} \mathrm{C}$, washed and imaged directly. The low temperature micrographs (Fig. S6 $\dagger$ ) present intensive cellular internalization, similarly with the result at $37^{\circ} \mathrm{C}$. This confirms that $6 \mathrm{P}$ and $10 \mathrm{P}$ are membrane permeable compounds and they enter

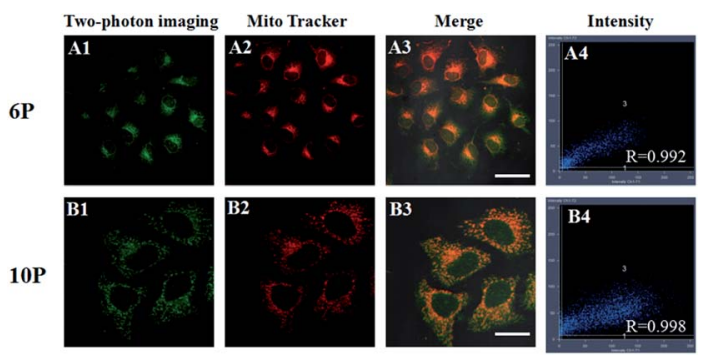

Fig. 7 (A1) Two-photon image of HepG2 cells incubated with $10 \mu \mathrm{M}$ $6 \mathrm{P}$ after $20 \mathrm{~min}$ of incubation, and washed with PBS buffer. $\lambda_{\mathrm{ex}}=$ $830 \mathrm{~nm}$ (emission wavelength from 550 to $650 \mathrm{~nm}$ ). (A2) One-photon image of HepG2 cells incubated with $10 \mu$ M MitoTracker ${ }^{\circledR}$ after 20 min of incubation and washed with PBS buffer. $\lambda_{\text {ex }}=633 \mathrm{~nm}$ (emission wavelength from 650 to $700 \mathrm{~nm}$ ). (A3) Merged image. (A4) Pearson's coefficient of HepG2 cells incubated with 6P. (B1) Two-photon image of HepG2 cells incubated with $10 \mu \mathrm{M} 10 \mathrm{P}$ after 20 min of incubation, and washed with PBS buffer. $\lambda_{\mathrm{ex}}=730 \mathrm{~nm}$ (emission wavelength from 550 to $650 \mathrm{~nm}$ ). (B2) One-photon image of HepG2 cells incubated with $10 \mu \mathrm{M}$ MitoTracker $\circledast$ after 20 min of incubation, and washed with PBS buffer. $\lambda_{\text {ex }}=633 \mathrm{~nm}$ (emission wavelength from 650 to $700 \mathrm{~nm}$ ). (B3) Merged image. (B4) Pearson's coefficient of HepG2 cells incubated with 10P. 


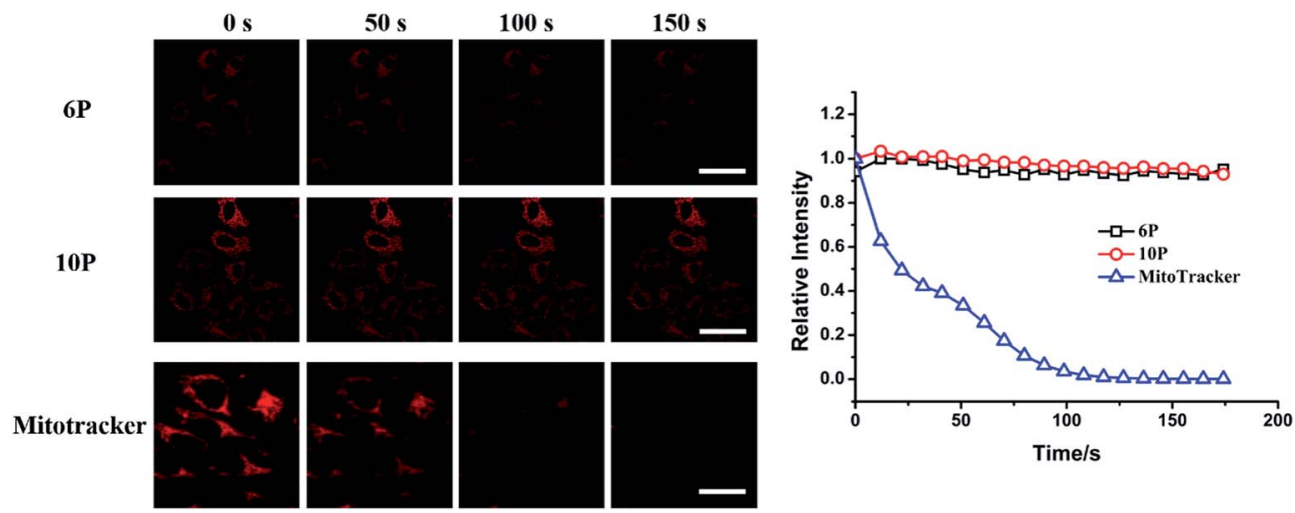

Fig. 8 Photon bleaching test of 6P and 10P in living cells under continuous laser irradiation.

cells via a temperature-independent pathway, possibly passive infusion rather than endocytosis, which is highly dependent on the temperature.

Photobleaching stability experiment. Photostability is one of the most important criteria for developing fluorescence imaging agents. The photostability of $6 \mathrm{P}$ and $10 \mathrm{P}$ were examined in comparison to the commercially available MitoTracker in living HepG2 cells via photobleaching experiments. As shown in Fig. 8, no significant changes in the fluorescence intensity of $6 \mathrm{P}$ and $10 \mathrm{P}$ can be observed after $150 \mathrm{~s}$. On the other hand, after successive irradiation at the same intensity, the fluorescence of MitoTracker is significantly bleached. The high photostability of $6 \mathrm{P}$ and $10 \mathrm{P}$ makes them favorable for long-term real-time tracking.

Internalization with larval zebrafish model. Since 6P and 10P were successfully utilized as a convenient and noninvasive 2PA probe in living cells with superb photostability and cell permeability, we were motivated to evaluate their potential in small animal labeling. Zebrafish ${ }^{30}$ was chosen and its larval

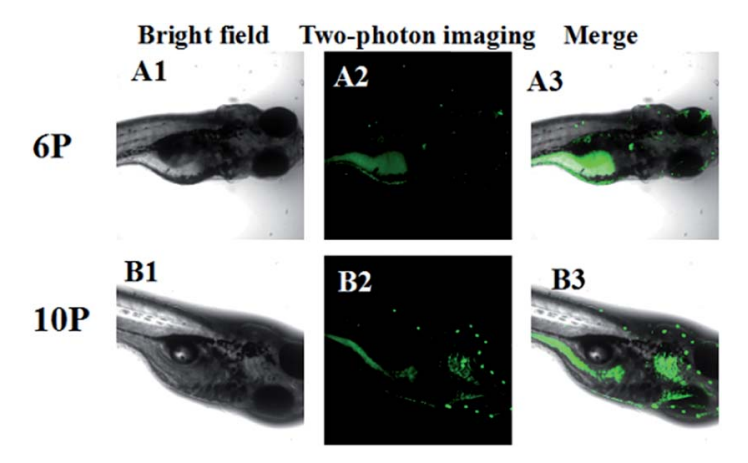

Fig. 9 (A1) Bright field image of $96 \mathrm{~h}$-zebrafish larva incubated with 10 $\mu \mathrm{M} 6 \mathrm{P}$ after $5 \mathrm{~h}$ of incubation at $28^{\circ} \mathrm{C}$ and washed with PBS buffer; (A2) two-photon image of $96 \mathrm{~h}$-zebrafish larva incubated with $10 \mu \mathrm{M} 6 \mathrm{P}$ after $5 \mathrm{~h}$ of incubation, and washed with PBS buffer. $\lambda_{\mathrm{ex}}=830 \mathrm{~nm}$ (emission wavelength from 550 to $650 \mathrm{~nm}$ ) and (A3) overlay of the bright field and 2PA images. (B1) Bright field image of $96 \mathrm{~h}$-zebrafish larvae incubated with $10 \mu \mathrm{M} 10 \mathrm{P}$ after $5 \mathrm{~h}$ of incubation at $28^{\circ} \mathrm{C}$, and washed with PBS buffer; (B2) two-photon image of $96 \mathrm{~h}$-zebrafish larva incubated with $10 \mu \mathrm{M} 10 \mathrm{P}$ after $5 \mathrm{~h}$ of incubation and washed with PBS buffer. $\lambda_{\text {ex }}=730 \mathrm{~nm}$ (emission wavelength from 550 to $650 \mathrm{~nm}$ ) and (B3) overlay of the bright field and 2PA images. The scale bars represent $100 \mu \mathrm{m}$. survival experiment was performed first to ensure the low cytotoxicity of $6 \mathrm{P}$ and $10 \mathrm{P}$ for living vertebrate animals. Subsequently $6 \mathrm{P}$ and 10P-labeled anaesthetized larval zebrafish was moved to TPFM to exam their precise location in the fish body under two-photon laser. As shown in Fig. 9, strong fluorescence can be observed in the intestine after five hours of incubation with $10 \mu \mathrm{M} 6 \mathrm{P}$ and $10 \mathrm{P}$ respectively. This result indicates that these two chromophores can be easily ingested by zebrafish in the water, and display good capability of living organism imaging using two-photon fluorescence microscopy, in accord with their good two-photon fluorescence properties.

Two-photon microscopy in zebrafish. All procedures involving animals were approved by and conformed to the guidelines of the Southwest University Animal Care Committee, College of Pharmaceutical Sciences. We have taken great efforts to reduce the number of animals used in these studies and also taken effort to reduce animal suffering from pain and discomfort.

\section{Conclusion}

In brief, two imidazole-pyrimidine hexafluorophosphate derivatives with the $\mathrm{D}-\pi-\mathrm{A}-\pi-\mathrm{D}$ configuration have been synthesized using a convenient method. Their photophysical properties can be tuned by a simple modification of the electron-donating and electron-withdrawing groups, which correlate both experimentally and theoretically. Relying on typical structural and comprehensive spectral data, the following structure-property relationships can be drawn: (1) 6P shows moderate 2PA cross sections in the near-IR region using 2PEF measurements, which indicates that intramolecular charge transfer (ICT) has a different effect on the intensity of the OPEF and 2PEF, and the $N, N$-bis(4-ethoxyphenyl)aniline groups as electron donor units should play a major role in the $2 \mathrm{PEF}$ intensity. (2) 10P exhibits satisfactory two-photon absorption in the near-infrared range around $730 \mathrm{~nm}$, with the introduction of ethoxyphenyl triphenylamine groups as auxiliary electron donors observed in a typical Z-scan measurement. (3) Importantly, the two unique imidazole-pyrimidine hexafluorophosphates as two-photon ratiometric probes can be used for quantifying and imaging organelles in living cells and 
tissues. Their advantages of exclusive mitochondria staining of living cells, high signal ratio, excitation with NIR light, good photostability, as well as low cytotoxicity at imaging concentrations, promise potential applications of $6 \mathrm{P}$ and $10 \mathrm{P}$ in biological and biomedical research.

\section{Acknowledgements}

This study was supported by the National Natural Science Foundation of China (51372003, 21501001, 51432001, 21602003, 51672002 and 51472002), Postdoctoral Science Foundation of Anhui Province (2016B092), Ministry of Education Funded Projects Focus on returned overseas scholar. The Higher Education Revitalization Plan Talent Project (2013), the Natural Science Foundation of Anhui Province (1508085MB34).

\section{References}

1 (a) L. Ji, Q. Fang, M.-S. Yuan, Z.-Q. Liu, Y.-X. Shen and H. F. Chen, Switching High Two-Photon Efficiency: From 3, 8, 13-Substituted Triindole Derivatives to Their 2, 7, 12Isomers, Org. Lett., 2010, 12, 5192-5195; (b) R. M. Edkins, L. J. Sewell, A. Beeby, A. S. Batsanov, K. Fucke, M. Drafz, J. A. K. Howard, O. Moutounet, F. Ibersiene, A. Boucekkine, E. Furet, Z. Q. Liu, J. F. Halet, C. Katan and T. B. Marder, Experimental and Theoretical Studies of Quadrupolar Oligothiophene-Cored Chromophores Containing Dimesitylboryl Moieties as $\pi$-Accepting End-Groups: Syntheses, Structures, Fluorescence, and One- and TwoPhoton Absorption, Chem.-Eur. J., 2014, 20, 13618-13635; (c) L. Ji, S. Griesbeck and T. B. Mader, Recent developments in and perspectives on three-coordinate boron materials: a bright future, Chem. Sci., 2017, 8, 846863.

2 X. C. Wang, X. H. Tian, Q. Zhang, P. P. Sun, J. Y. Wu, H. P. Zhou, B. K. Jin, J. X. Yang, S. Y. Zhang, C. K. Wang, X. T. Tao, M. H. Jiang and Y. P. Tian, Assembly, TwoPhoton Absorption and Bioimaging Applications of A Cuprous Cluster, Chem. Mater., 2010, 24, 954-961.

3 Z. D. Liu, R. L. Zhang, Q. Zhang, H. J. Ding, C. K. Wang and S. L. Li, Crystal structures, photophysical properties and significantly different two-photon excited fluorescence of the trans- and cis-oligo(phenylene vinylene), RSC Adv., 2014, 4, 2620-2623.

4 X. L. Yue, C. O. Yanez, S. Yao and K. D. Belfield, Selective Cell Death by Photochemically Induced $\mathrm{pH}$ Imbalance in Cancer Cells, J. Am. Chem. Soc., 2013, 135, 2112-2115.

5 D. X. Li, X. Sun, J. M. Huang, Q. Wang, Y. Feng and M. Chen, A carbazole-based "turn-on" two-photon fluorescent probe for biological $\mathrm{Cu}^{2+}$ detection vis $\mathrm{Cu}^{2+}$-promoted hydrolysis, Dyes Pigm., 2016, 125, 185-191.

6 (a) A. R. Sarkar, D. E. Kang, M. K. Hwan and B. R. Cho, TwoPhoton Fluorescent Probes for Metal Ions in Live Tissues, Inorg. Chem., 2014, 53, 1749-1803; (b) D. X. Li, X. Sun, J. M. Huang, Q. Wang, Y. Feng and M. Chen, A mitochondria-targeted two-photon fluorescent probe for highly selective and rapid detection of hypochlorite and its bio-imaging in living cells, Sens. Actuators, B, 2016, 222, 483-491; (c) S. Yao and K. D. Belfield, Two-Photon Fluorescent Probes for Bioimaging, Eur. J. Org. Chem., 2012, 17, 3199-3217.

7 L. Yuan, L. Wang, B. K. Agrawalla, S. J. Park, H. Zhu and B. Sivaraman, Development of Targetable Two-Photon Fluorescent Probes to Image Hypochlorous Acid in Mitochondria and Lysosome in Live Cell and Inflamed Mouse Model, J. Am. Chem. Soc., 2015, 137, 5930-5938.

8 T. T. Zou, C. N. Lok, Y. M. E. Fung and C. C. Che, Luminescent organoplatinum(II) complexes containing bis(N-heterocyclic carbene) ligands selectively target the endoplasmic reticulum and induce potent photo-toxicity, Chem. Commun., 2013, 49, 5423-5425.

9 H. Zhang, J. L. Fan, J. Y. Wang, S. Z. Zhang, B. R. Dou and X. J. Peng, An Off-On $\mathrm{CO}_{X-2}$-Specific Fluorescent Probe: Targeting the Golgi Apparatus of Cancer Cells, J. Am. Chem. Soc., 2013, 135, 11663-11669.

10 S. H. Jung, J. Y. Han, J. H. Lee, J. H. Lee, J. M. Choi and H. S. Kweon, Enhanced NIR Radiation-Triggered Hyperthermia by Mitochondrial Targeting, J. Am. Chem. Soc., 2015, 137, 3017-3023.

11 (a) B. Liu, H. L. Zhang, J. Liu, Y. D. Zhao, Q. M. Luo and Z. L. Huang, Novel pyrimidine-based amphiphilic molecules: synthesis, spectroscopic properties and applications in two-photon fluorescence microscopic imaging, J. Mater. Chem., 2007, 17, 2921-2929; (b) H. Akdas-Kilig, T. Roisnel, I. Ledoux and H. L. Bozec, A new class of bipyrimidine-based octupolar chromophores: synthesis, fluorescent and quadratic nonlinear optical properties, New J. Chem., 2009, 33, 1470-1473.

12 (a) Z. J. Liu, T. Chen, B. Liu, Z. L. Huang, T. Huang and S. Y. Li, Two-photon absorption of a series of V-shape molecules: the influence of acceptor's strength on twophoton absorption in a noncentrosymmetric $\mathrm{D}-\pi-\mathrm{A}-\pi-\mathrm{D}$ system, J. Mater. Chem., 2007, 17, 4685-4689; (b) S. Achelle and N. Ple, Pyrimidine Ring as Building Block for the Synthesis of Functionalized $\pi$-Conjugated Materials, Curr. Org. Synth., 2012, 9, 163-187.

13 (a) Z. J. Liu, P. Shao, Z. L. Huang, B. Liu, T. Chen and J. G. Qin, Two-photon absorption enhancement induced by aggregation due to intermolecular hydrogen bonding in Vshaped 2-hydroxypyrimidine derivatives, Chem. Commun., 2008, 2260-2262; (b) L. Li, Y. P. Tian, J. X. Yang, P. P. Sun, L. Kong and J. Y. Wu, Two-photon absorption enhancement induced by aggregation with accurate photophysical data: spontaneous accumulation of dye in silica nanoparticles, Chem. Commun., 2010, 46, 1673-1675; (c) J. P. Malval, S. Achelle, L. Bodiou, g. A. OPAngenber, L. C. Gomez, O. Soppera and F. R. Guen, Two-photon absorption in conformationally twisted $\mathrm{D}-\pi-\mathrm{A}$ oligomer: a synergic photosensitizing approach for multiphoton lithography, J. Mater. Chem. C, 2014, 2, 7869-7880.

14 (a) X. Y. Zhou, S. Khanapur, A. P. Huizing, R. Zijlma, M. Schepers, R. A. J. O. Dierckx, A. V. Waarde, E. F. J. D. Vries and P. H. Elsinga, Synthesis and Preclinical Evaluation of 2-(2-Furanyl)-7-[2-[4-[4-(2-[11 C]methoxyethoxy) 
phenyl]-1-piperazinyl]ethyl]7H-pyrazolo[4,3-e][1,2,4]triazolo [1,5-c]pyrimidine-5-amine ([11C]preladenant) as a PET Tracer for the Imaging of Cerebral Adenosine A2A Receptors, J. Med. Chem., 2014, 57, 9204-9210; (b) L. Y. Ma, Y. C. Zheng, S. Q. Wang, B. Wang, Z. R. Wang, L. P. Pang, M. Zhang, J. W. Wang, L. Ding, J. Li, C. Wang, B. Hu, Y. Liu, X. D. Zhang, J. J. Wang, Z. J. Wang, W. Zhao and H. M. Liu, Synthesis, and Structure Activity Relationship of Novel LSD1 Inhibitors Based on Pyrimidine-Thiourea Hybrids As Potent, Orally Active Antitumor Agents, J. Med. Chem., 2015, 58, 1705-1716.

15 Q. Zhang, X. H. Tian, Z. J. Hu, C. Brommesson, J. Y. Wu, H. P. Zhou, J. X. Yang, Z. Q. Sun, Y. P. Tian and K. Uvdal, Nonlinear optical response and two-photon biological applications of a new family of imidazole-pyrimidine derivatives, Dyes Pigm., 2016, 126, 286-295.

16 Q. Zhang, L. Luo, H. Xu, Z. J. Hu, C. Brommesson, J. Y. Wu, Z. Q. Sun, Y. P. Tian and K. Uvdal, Design; synthesis, linear and nonlinear photophysical properties of novel pyrimidinebased imidazole derivatives, New J. Chem., 2016, 40, 34563463.

17 W. C. Xu, J. R. Zuo, L. L. Wang, J. N. Ji and H. Chao, Dinuclear ruthenium(II) polypyridyl complexes as single and two-photon luminescence cellular imaging probes, Chem. Commun., 2014, 50, 2123-2125.

18 V. W. W. Yam, R. P. L. Tang, K. M. C. Wong, X. X. Lu, K. K. Cheung and N. Y. Zhu, Syntheses, Electronic Absorption, Emission, and Ion-Binding Studies of Platinum(II) $\mathrm{C}^{\wedge} \mathrm{N}^{\wedge} \mathrm{C}$ and Terpyridyl Complexes Containing Crown Ether Pendants, Chem.-Eur. J., 2002, 8, 4066-4076.

19 Y. W. Shi, M. M. Shi, J. C. Huang, H. Z. Chen, M. Wang, X. D. Liu, Y. G. Ma, H. Xu and B. Yang, Fluorinated Alq derivatives with tunable optical properties, Chem. Commun., 2006, 1941-1943.

20 D. G. Chen, C. Zhong, X. H. Dong, Z. L. Liu and J. G. Qin, A new building block, bis(thiophene vinyl)-pyrimidine, for constructing excellent two-photon absorption materials: synthesis, crystal structure and properties, J. Mater. Chem., 2012, 22, 4343-4348.

21 S. C. F. Kui, S. S. Y. Chui, C. M. Che and N. Y. Zhu, Structures, Photoluminescence, and Reversible Vapoluminescence Properties of Neutral Platinum(II) Complexes Containing Extended $\pi$-Conjugated Cyclometalated Ligands, J. Am. Chem. Soc., 2006, 128, 8297-8309.
22 Y. Ren, X.-Q. Yu, D.-J. Zhang, D. Wang, M.-L. Zhang, G.-B. Xu, X. Zhao, Y.-P. Tian, Z.-S. Shao and M.-H. Jiang, Synthesis, structure and properties of a new two-photon photopolymerization initiator, J. Mater. Chem., 2002, 12, 3431-3437.

$23 \mathrm{~F} . \mathrm{Xu}, \mathrm{Z}$. W. Wang and Q. H. Gong, Synthesis, characterization, and optical properties of two-photonabsorbing octupolar molecule with an $s$-triazine core, opt. Mater., 2007, 29, 723-727.

24 L. Li, Y. P. Tian, J. X. Yang, P. P. Sun, J. Y. Wu, H. P. Zhou, S. Y. Zhang, B. K. Jin, X. J. Xing, C. K. Wang, M. Li, G. H. Cheng, H. H. Tang, W. H. Huang, X. T. Tao and M. H. Jiang, Facile Synthesis and Systematic Investigations of a Series of Novel Bent-Shaped Two-Photon Absorption Chromophores Based on Pyrimidine, Chem.-Asian J., 2009, 4, 668-680.

25 L. Li, Y. P. Tian, J. X. Yang, P. P. Sun, L. Kong, J. Y. Wu, H. P. Zhou, S. Y. Zhang, B. K. Jin, X. T. Tao and M. H. Jiang, Two-photon absorption enhancement induced by aggregation with accurate photophysical data: spontaneous accumulation of dye in silica nanoparticles, Chem. Commun., 2010, 46, 1673-1675.

26 H. Y. Woo, B. Liu, B. Kohler, D. Korystov, A. Mikhailovsky and G. C. Bazan, Solvent effects on the two-photon absorption of distyrylbenzene chromophores, J. Am. Chem. Soc., 2005, 127, 14721-14729.

27 X. C. Wang, X. H. Tian, Q. Zhang, Y. P. Tian, P. P. Sun and J. Y. Wu, Assembly, twophoton absorption, and bioimaging of living cells of a cuprous cluster, Chem. Mater., 2012, 24, 954-961.

28 J. J. Shao, Z. P. Guan, Y. L. Yan, C. J. Jiao, Q. H. Xu and C. Y. Chi, Synthesis and characterizations of star-shaped octupolar triazatruxenes-based two-photon absorption chromophores, J. Org. Chem., 2011, 76, 780-790.

29 Z. Q. Ji, Y. J. Li, T. M. Pritchett, N. S. Makarov, J. E. Haley, Z. J. Li, M. Drobizhev, A. Rebane and W. F. Sun, OnePhoton Photophysics and Two-Photon Absorption of 4-[9,9Di(2-ethylhexyl)-7-diphenylaminofluoren-2-yl]-2,2':6', $2^{\prime \prime}$ terpyridine and Their Platinum Chloride Complexes, Chem.Eur. J., 2011, 17, 2479-2491.

30 S. K. Ko, X. Chen, J. Yoon and I. Shin, Zebrafish as a good vertebrate model for molecular imaging using fluorescent probes, Chem. Soc. Rev., 2011, 40, 2120-2130. 